\title{
Prevalencia y detección de la desnutrición en pacientes en diálisis en la unidad de nefrología del Hospital General de Segovia
}

\section{Carmen Gutiérrez Martín - Azucena Mayoral Peñas - Sonia Velasco Ballestero}

Unidad de Diálisis. Hospital General de Segovia

\section{Resumen}

La malnutrición es uno de los mayores predictores de mortalidad de pacientes en diálisis. La inflamación, el hipercatabolismo asociado al procedimiento dialítico, las situaciones comórbidas y la reducción en la ingesta calórica y proteica son causas de malnutrición en estos pacientes.

La disponibilidad de un marcador nutricional ideal (sensible, fácil de medir y reproducible) es difícil debido a los cambios metabólicos asociados a la insuficiencia renal. Para conocer el estado nutricional de los pacientes y hacer recomendaciones individualizadas, es necesario utilizar un conjunto de medidas antropométricas, bioquímicas y dietéticas; por otro lado el análisis del vector obtenido mediante bioimpedancia ha sido validado para su empleo en estos pacientes y podría ayudar en la detección temprana de alteraciones nutricionales.

En este estudio evaluamos la prevalencia de malnutrición en 43 pacientes en programa de diálisis usando parámetros antropométricos y bioquímicos clásicos, así como encuestas dietéticas y análisis de bioimpedancia vectorial. Según parámetros bioquímicos entre un $41,9 \%$ al 53\% de nuestros pacientes presentaban malnutrición; al utilizar parámetros antropométricos solamente el $26 \%$ estaba malnutrido. El análisis por bioimpedancia demostró que el $18,6 \%$ de ellos mostraban una reducción en su masa corporal total.

\author{
Correspondencia: \\ Carmen Gutiérrez Martín \\ Unidad de Diálisis \\ Hospital General de Segovia \\ Crta. de Ávila s/n-40002 Segovia \\ cgutierrez@hgse.sacyl.es
}

Los parámetros antropométricos y de bioimpedancia se encontraban dentro del rango normal en la mayoría de los pacientes. Los pacientes con una adecuada ingesta proteico-calórica no mostraron mejores parámetros nutricionales que el resto en términos bioquímicos y de composición corporal. Por ello creemos que más que un problema de ingesta, la malnutrición en estos pacientes es resultado de inflamación e hipercatabolismo.

\section{Prevalence and detection of malnutrition in patients on dialysis in the General Hospital of Segovia}

\section{Abstract}

Malnutrition is one the most powerful independent predictors of morbimortality in patients on chronic dialysis. Inflammation, hypercatabolism associated to the dialysis procedure, comorbidities and reductions in protein and calories ingestion are though to be the main causes of malnutrition in those patients.

The availability of an ideal (sensible, easy to measure, and reproducible) malnutrition marker, is difficulties by the metabolic changes associated to chronic renal failure. To know the nutritional sta- 
tus of a patient, in order to make individual nutritional recommendations, it is necessary to use a panel of anthropometric, biochemical and dietary measurements; on the other hand, bioelectrical impedance vector analysis has been validated for its use in patients on hemo and peritoneal dialysis and might be helpful for the early detection of nutritional alterations.

In this study we evaluated the prevalence of malnutrition in 43 stable dialysis patients, using classical biochemical and anthropometrics parameters, as well as dietary surveys, and bioelectrical impedance vector analysis. According to biochemical parameters, $41,9 \%$ to $53 \%$ of our patients showed malnutrition, whereas using anthropometrics parameters only $26 \%$ were malnourished. Bioelectrical impedance vector analysis demonstrated that $18,6 \%$ of patients showed a reduction on total body fat. Anthropometric and bioimpedance muscle parameters were within normal range in most patients. Patients with an adequate protein-calorie ingestion did not showed better nutritional parameters than the rest, in terms of biochemical and body composition values, so we think that more than a problem of ingestion, malnutrition in these patients is the result of inflammation and hypercatabolism.

\section{KEY WORDS:}

\section{- NUTRITION}

- DIALYSIS

- CALORIC INGESTION

- PROTEIN INGESTION

- ANTHROPOMETRIC PARAMETERS

BIOELECTRICAL IMPEDANCE VECTOR ANALYSIS

\section{Introducción}

El estado nutricional es uno de los mayores predictores de supervivencia en los pacientes en hemodiálisis (HD) y en diálisis peritoneal (DP). Cierto grado de desnutrición es muy frecuente $(20-80 \%)$ en los pacientes con enfermedad renal crónica $(E R C)^{1-3}$ y se ha asociado con una elevada morbimortalidad ${ }^{4,5}$. La monitorización frecuente del estado nutricional, identificando precozmente el déficit alimentario y las alteraciones de la composición corporal, es fundamental para mejorar los resultados en estos pacientes. Se han utilizado múltiples parámetros para medir el estado de nutrición en diálisis, pero todos ellos adolecen de diferentes grados de inexactitud o sesgos derivados de las complicaciones que se producen en la ERC. El estado de sobrehidratación altera los datos antropométricos y provoca hemodilución de otros parámetros nutricionales clásicos como la albúmina. La inflamación, el hipercatabolismo y las situaciones comórbidas que a menudo tienen estos pacientes, también pueden influir de forma negativa en la disminución de proteínas y albúmina ${ }^{6}$ Éstas y otras alteraciones asociadas a la ERC, hacen muy difícil disponer de un marcador ideal de malnutrición que sea sensible, fácil de medir, y reproducible. Según las guías de nutrición tendremos por tanto que utilizar un panel de medidas antropométricas, bioquímicas y de ingesta para determinar de manera individualizada el estado de nutrición y poder hacer las correspondientes recomendaciones dietéticas ${ }^{7}$.

El análisis del estado nutricional y de la composición corporal mediante impedancia bioeléctrica (BIA) convencional ${ }^{8}$ ha tenido múltiples problemas para su aplicación práctica en los pacientes en diálisis dado que puede dar estimaciones no fiables en pacientes con hidratación anómala. La aparición de la bioimpedancia vectorial (BIVA) donde el vector impedancia $Z$ es representado en coordenadas polares y el vector medido en el individuo se confronta con la distribución de los vectores de una población sana de referencia ( 726 sujetos italianos sanos con edades entre 18-85 años), con tres percentíles de referencia (elipses de tolerancia al $50 \%, 75 \%$ y $95 \%$ ) y específica por géner $0^{9}$ ha sido validada para su utilización en pacientes en DP y $\mathrm{HD}^{10,11}$ y podría ayudarnos para detectar precozmente las alteraciones nutricionales y de la composición corporal.

El descenso de la ingesta calórica y proteica y el catabolismo asociado al procedimiento hemodialítico se han considerado como la principal causa de desnutrición en pacientes en HD ${ }^{4,5}$. Las guías de nutrición ${ }^{7}$ recomiendan para estos pacientes la ingesta, como mínimo, de 1,2 gr/kg de proteína y 30-35 Kcal / kg / día. No obstante, existen muy pocos estudios donde se realicen encuestas dietéticas en pacientes en diálisis y se demuestre que la desnutrición se debe a déficit en la ingesta ${ }^{2}$. 


\section{Objetivos}

El primer objetivo fue analizar la prevalencia de desnutrición en nuestros pacientes en diálisis (DP y HD) y evaluar los diferentes métodos para detectarla, analizando lo que aportan nuevas técnicas como la BIVA sobre los parámetros que clásicamente se han utilizado en nuestra Unidad.

Un segundo objetivo fue ver si los pacientes con ingestas adecuadas tenían mejores parámetros de nutrición en términos bioquímicos y de composición corporal.

\section{Pacientes y Métodos}

Se incluyeron en el estudio todos los pacientes en diálisis (DP y HD) en el H. General de Segovia en el mes de abril 2008. Se excluyeron aquellos pacientes que se encontraban hospitalizados por un proceso agudo en el momento del inicio del estudio o los que estaban recibiendo suplementos nutricionales.

\section{Se realizó un corte trasversal donde se determina-} ron:

1. El promedio de las ingesta calórica y proteica diarias corregidas para el peso ideal del paciente, mediante encuesta dietética de 3 días (programa informático Dietsource $3.0 \circledR$ Novartis). También se registró el porcentaje de lípidos e hidratos de carbono, y las ingestas de sodio, potasio y fósforo.

2. Los siguientes parámetros bioquímicos y hematológicos: Albúmina, Prealbúmina, Transferrina, Colesterol, Hematocrito, Creatinina, $\mathrm{pH}$ y bicarbonato, proteína $\mathrm{C}$ reactiva, mediante los métodos rutinarios del H. General de Segovia. La analítica se realizó previa a la primera hemodiálisis de la semana; los pacientes en turno de tarde fueron instruidos para permanecer en ayunas al menos 4 horas antes de la extracción. En los pacientes en DP las extracciones se realizaron en el turno de mañana en ayunas.

3. La dosis de diálisis se midió mediante el cálculo del KT/V y se estimó la ingesta proteica a partir de la tasa de catabolismo proteico normalizada para el peso actual (nPCR).

4. Las medidas antropométricas, se realizaron post hemodiálisis en el día intermedio de la semana usando técnicas estándar ${ }^{12}$ e incluían: talla, peso,
BMI (ó IMC: índice de masa corporal), pliegue graso tricipital (PGT), circunferencia de brazo (CB), perímetro muscular de brazo (PMB), circunferencias de muñeca, cintura y cadera. Los resultados se estandarizaron calculando en cada caso el porcentaje respecto al percentil 50 de datos obtenidos para una población de adultos españoles normales de la misma edad y sexo $0^{13,14}$, considerando déficit moderados o severos los superiores al $80 \%$ de la población normal. La relación cintura-cadera (RCC), fue determinada y se estratificó el riesgo cardiovascular de acuerdo con las recomendaciones de la Sociedad Española de Endocrinología y Comité de Expertos de la $0 \mathrm{MS}^{15,16}$, considerando que en varones existen 4 tipos de riesgos (bajo 0.83-0.88, moderado 0.88-0.95, alto 0.95-1.01 y muy alto $>1.01$ ) y en mujeres 3 riesgos (bajo 0.72-0.75, moderado $0.78-0.82$ y alto $>0.82$ ). En los pacientes en DP se realizaron mediciones el día de la analítica.

5. BIVA post-diálisis en el día intermedio de la semana, y en pacientes en DP con abdomen vacío, (Vectorial BIA 101; Akern, Florence, Italy) midiendo resistencia $(\mathrm{R})$ reactancia $\left(\mathrm{Xc}_{\mathrm{c}}\right)$, ángulo de fase (PA), intercambio sodio-potasio (I Na/K), agua total (TBW), agua extracelular (ECW), agua intracelular (ICW), masa celular, índice de masa celular (masa celular/talla²) (BCM), masa grasa (FM), masa libre de grasa (FFM) y masa muscular. Estos datos se compararon con los valores normales dados para personas de su misma edad y peso $0^{9}$. Hay que tener en cuenta que disponemos de valores normales hasta los 70 años, tanto para los datos antropométricos clásicos como para la bioimpedancia; por tanto en los pacientes mayores de 70 años decidimos considerar el valor normal del que disponemos para 70 años.

El análisis estadístico se realizó con el programa SPSS. Los valores se expresan como porcentajes 0 medias \pm SD. Se consideró como significación estadística un valor de $p<0.05$. Utilizamos como medida de comorbilidad el índice de Charlson modificado según lo descrito por Beddhu ${ }^{17}$. Utilizamos el test de $\mathrm{Chi}^{2}$ para comparar proporciones, la t-Student para comparar medias; el test de Pearson para establecer coeficientes de correlación lineales o el de Sperman si alguna variable era ordinal o no tenía una distribución normal. 


\section{Resultados}

Se incluyeron en el estudio un total de 43 pacientes (32 en HD y 11 en DP) con una edad media de $71,7 \pm 10,4$ y con un tiempo medio en diálisis de $47 \pm 46$ meses (rango entre 1 y 210 meses).10 pacientes $(23,3 \%$ eran diabéticos) y el porcentaje de varones era del $55,8 \%$. No existían diferencias de edad entre los pacientes en HD y DP $(71,5 \pm 11,5 \mathrm{vs}$ $72,3 \pm 7,1 \mathrm{~ns})$, ni en el índice de Charlson. Los pacientes en HD llevaban más tiempo en diálisis que los de DP $(32 \pm 43,7$ vs $24,7 \pm 21 p=0,009)$.

En la tabla 1 aparecen detallados los datos analíticos y antropométricos del grupo global y la comparación entre HD y DP. Como podemos observar, los pacientes en DP tienen en general todas las medidas antropométricas más altas que los pacientes en HD con excepción del PGT, perímetro muscular del brazo y perímetro de muñeca. Utilizando criterios analíticos de malnutrición: 23 pacientes $(53,5 \%)$ tenían una albúmina menor de 3,8 $\mathrm{gr} / \mathrm{dl}, 18$ pacientes (41,9\%) tenían prealbumina menor de $30 \mathrm{mg} / \mathrm{dl}$ y sólo 2 pacientes $(4,7 \%)$ tenían el colesterol menor de $100 \mathrm{mg} /$ dl. Utilizando criterios antropométricos clásicos: 14 $(32,6 \%$ ) pacientes (12 en HD y 2 en DP) presentaban una desnutrición calórica moderada-severa por disminución del PGT. Sólo $3(7 \%)$ y $1(2,3 \%)$ pa- cientes, tenían una disminución moderada-severa del CB y $\mathrm{PMB}$ respectivamente.

En cuanto al BMI, 11 pacientes $(26,2 \%)$ presentaban $\mathrm{BMI}<23$, cifra que en la literatura se ha asociado a mayor mortalidad en diálisis7,18. Cuando comparamos los pacientes con $\mathrm{BMI}<23$ frente al resto observamos que todos los pacientes con BMI bajo estaban en HD: estos pacientes tenían menos grasa medida tanto con bioimpedancia como porcentaje de percentil 50 sobre pliegue graso tricipital, perímetro de cintura y cadera (tabla 2) pero no había diferencias analíticas ni en ingesta calórica ni proteica corregidas para peso ideal. EI BMI presentó una fuerte correlación con la grasa medida por bioimpedancia $(R=0.80 p=0,000)$ y con el perímetro de cintura ( $R=0,68 p=0,000)$ y más débil con el $P G T(R=0,40$ $p=0,07)$, no alcanzando la significación estadística con el percentil 50 del PGT ( $R=0,23$ ns).

En cuanto a la RCC no observamos diferencias significativas entre ambos sexos, siendo ligeramente más alto en varones ( 0.97 frente a $0.93 \mathrm{~ns}$ ). No encontramos asociación entre el RCC y el PGT, la ingesta de calorías o el porcentaje de grasa medida por BIVA. Un $72,1 \%$ de los pacientes tendrían obesidad abdominal con alto riesgo cardiovascular medido por RCC; sólo 3 pacientes ( $7 \%$ ) tienen un RCC bajo.

\begin{tabular}{|l|c|c|c|c|}
\hline & Total $\mathbf{n = 4 3}$ & HD $\mathbf{n = 3 2}$ & DP $\mathbf{n = 1 1}$ & $\mathbf{P}$ HD vs DP \\
\hline Albúmina & $3,67 \pm 0,33$ & $3,7 \pm 0,32$ & $3,5 \pm 0,31$ & $\mathrm{~ns}$ \\
\hline Prealbúmina & $32,85 \pm 9,6$ & $31,6 \pm 9,5$ & $36,4 \pm 9,5$ & $\mathrm{~ns}$ \\
\hline Colesterol & $147 \pm 30$ & $146 \pm 30,1$ & $151 \pm 33$ & $\mathrm{~ns}$ \\
\hline \% Peso ideal & $116,8 \pm 22$ & $110,8 \pm 20$ & $134,4 \pm 18$ & $\mathrm{P}=0.001$ \\
\hline Peso real & $63 \pm 11,6$ & $59,7 \pm 10,7$ & $72,4 \pm 8,7$ & $0=0.001$ \\
\hline Talla & $1,55 \pm 0,1$ & $1,55 \pm 0,1$ & $1,56 \pm 0,1$ & $\mathrm{~ns}$ \\
\hline Superficie corporal & $1,62 \pm 0,18$ & $1,58 \pm 0,17$ & $1,74 \pm 0,14$ & $\mathrm{P}=0.008$ \\
\hline BMI & $25,9 \pm 4,55$ & $24,5 \pm 4,1$ & $29,9 \pm 3,39$ & $\mathrm{P}=0,000$ \\
\hline PGT & $12,9 \pm 4,9$ & $12,6 \pm 4,9$ & $13,7 \pm 5,2$ & $\mathrm{~ns}$ \\
\hline \%percentil50 PGT & $93,5 \pm 29$ & $91,14 \pm 31,4$ & $100,4 \pm 20,6$ & $\mathrm{~ns}$ \\
\hline CB & $27,3 \pm 4,1$ & $26,4 \pm 4,1$ & $29,9 \pm 3,39$ & $\mathrm{P}=0.016$ \\
\hline \%percentil50 CB & $105,5 \pm 16,6$ & $101,5 \pm 15,9$ & $116,8 \pm 13,1$ & $\mathrm{P}=0,007$ \\
\hline PMB & $23,35 \pm 3,8$ & $22,8 \pm 4,1$ & $24,1 \pm 2$ & $\mathrm{~ns}$ \\
\hline \% percentil50 PMB & $109,8 \pm 18,5$ & $107,4 \pm$ & $116,8 \pm 12,5$ & $\mathrm{~ns}$ \\
\hline Perímetro cadera & $101 \pm 10$ & $99,4 \pm 10,2$ & $105,7 \pm 8,5$ & $\mathrm{P}=0,07$ \\
\hline Perímetro cintura & $96,3 \pm 12,6$ & $93,7 \pm 13,3$ & $104 \pm 5,8$ & $\mathrm{P}=0.001$ \\
\hline Perímetro muñeca & $17 \pm 1,3$ & $17 \pm 1,3$ & $17,1 \pm 1,5$ & $\mathrm{~ns}$ \\
\hline RCC & $0,95 \pm 0,1$ & $0,94 \pm 0,1$ & $0,98 \pm 0,08$ & $\mathrm{~ns}$ \\
\hline
\end{tabular}

BMI= body mass index,

PGT= pliegue graso tricipital,

$\mathbf{C B}=$ circunferencia de brazo,

$\mathbf{P M B}=$ perímetro muscular del brazo,

$\mathbf{R C C}=$ relación cintura cadera .

Tabla 1. Datos antropométricos y analíticos globales y comparación HD vs DP
Según encuesta: 19 de los 43 pacientes (44,2\%) ingerían menos de $30 \mathrm{Kcal}$ /Kgr de peso ideal al día, y 6 (14\%) menor de $25 \mathrm{Kcal} / \mathrm{Kg} /$ día: estos pacientes no presentaban diferencias ni analíticas ni antropométricas con los pacientes que ingerían más de $30 \mathrm{Kcal}$ /peso. Cuando comparamos los 13 pacientes con ingestas superiores a 35 $\mathrm{Kcal} /$ peso frente al resto, vimos que tenían menor edad $(65,4 \pm 15$ vs $74,5 \pm 6$ $\mathrm{p}=0,07$ ) y menor comorbilidad medida por índice de Charlson $(6,5 \pm 2$ vs 8,5 $\pm 1 p=0.000$ ). En cuanto a la composición corporal tampoco encontramos diferencias en los parámetros antropométricos clásicos, si bien los pacientes con ingesta superiores a $35 \mathrm{Kcal} / \mathrm{kg}$ tenían mayor porcentaje de masa celular ( $51 \pm 7$ vs $40,3 \pm 9 p=0,001)$ y de masa muscular $(44,9 \pm$ vs $37 \pm 7 p=0,018)$ con 


\begin{tabular}{|l|c|c|c|}
\hline & $\begin{array}{c}\text { BMI <=23 } \\
\mathbf{( N = 1 2 )}\end{array}$ & $\begin{array}{c}\text { BMI }>\mathbf{2 3} \\
\mathbf{( N = 3 1 )}\end{array}$ & $\mathbf{P}$ \\
\hline Colesterol (mg/dl) & $153,6 \pm 32$ & $144,8 \pm 30$ & $\mathrm{~ns}$ \\
\hline Albúmina (g/dl) & $3,6 \pm 0,3$ & $3,6 \pm 0,4$ & $\mathrm{~ns}$ \\
\hline Transferrina & $168 \pm 26$ & $185 \pm 39$ & $\mathrm{~ns}$ \\
\hline Prealbúmina & $31,8 \pm 10$ & $32,3 \pm 11$ & $\mathrm{~ns}$ \\
\hline H0MA & $2,5 \pm 1,8$ & $4,9 \pm 3,9$ & 0,047 \\
\hline$\%$ Diabéticos & 16,7 & 25,8 & $\mathrm{~ns}$ \\
\hline Kcal/peso ideal & $32,6 \pm 9,7$ & $30,7 \pm 6,7$ & $\mathrm{~ns}$ \\
\hline $\begin{array}{l}\text { Proteína } \\
\text { (gr)/peso ideal }\end{array}$ & $1,2 \pm 0,3$ & $1,4 \pm 0,3$ & $\mathrm{~ns}$ \\
\hline Masa grasa(Kg) & $38 \pm 6,5$ & $46,9 \pm 8,2$ & 0,00 \\
\hline Masa grasa(\% basal) & $25 \pm 10$ & $31,6 \pm 8$ & 0,003 \\
\hline Masa celular (Kg) & $15,7 \pm 8$ & $21,2 \pm 7$ & 0,038 \\
\hline $\begin{array}{l}\text { Masa celular } \\
\text { en porcentaje }\end{array}$ & $40 \pm 8,8$ & $44,5 \pm 13,1$ & $\mathrm{~ns}$ \\
\hline Masa muscular (\%) & $40 \pm 14$ & $39,1 \pm 8$ & $\mathrm{~ns}$ \\
\hline $\begin{array}{l}\text { Masa muscular } \\
\text { (Kg/ talla2) }\end{array}$ & $8,5 \pm 3,4$ & $10,9 \pm 2,2$ & 0,001 \\
\hline $\begin{array}{l}\% \text { P50 Pliegue } \\
\text { tricipital }\end{array}$ & $74,2 \pm 24$ & $101,4 \pm 27$ & 0,005 \\
\hline $\begin{array}{l}\text { \% P50 } \\
\text { Circunferencia brazo }\end{array}$ & $92,7 \pm 14$ & $110,6 \pm 15$ & $\mathrm{~ns}$ \\
\hline $\begin{array}{l}\text { \%P50 perímetro } \\
\text { muscular }\end{array}$ & $102,6 \pm 10$ & $112,8 \pm 20$ & $\mathrm{~ns}$ \\
\hline Perímetro de muñeca & $16,3 \pm 1,2$ & $17,3 \pm 1,3$ & 0,027 \\
\hline Perímetro cadera & $92,5 \pm 7$ & $104,3 \pm 9$ & 0,000 \\
\hline Perímetro de cintura & $84 \pm 10$ & $101 \pm 10$ & 0,000 \\
\hline Sexo (\% varones) & $41,7 \%$ & $58,3 \%$ & $\mathrm{~ns}$ \\
\hline
\end{tabular}

Tabla 2. Comparación pacientes con BMI mayor o menor de $23 \mathrm{Kg} / \mathrm{m}^{2}$

mayor PA $(5,6$ vs $3,9 p=0,001)$ y menor intercambio $\mathrm{Na} / \mathrm{K}(1 \pm 0,2$ vs $1,6 \pm 0,7 \mathrm{p}=0,026)$.

En la analítica únicamente se observa que los pacientes con mayor ingesta tienen ureas más altas (156 \pm 37 vs $129 \pm 36 p=0,029)$ y niveles más bajos de bicarbonato $(21,2 \pm 2$ vs $24,7 \pm 3,7 p=0,003)$. En trece pacientes $(30,2 \%)$ la ingesta proteica era menor de 1,2 gr/kg y sólo un paciente tenía una ingesta proteica inferior a 0,8 gr/kg. Cuando comparamos a los 30 pacientes con ingesta igual o superior a 1,2 gr con el resto, las únicas diferencias significativas fueron una urea menor $(121,5 \pm 26,7$ vs $144,3 \pm 41,3 \mathrm{p}=0,038)$ y menor $\mathrm{pH}(7,37 \pm 0,05$ vs $7,33 \pm 0,06 p=0,02)$, sin diferencias antropométricas ni por bioimpedancia.

Los datos de la bioimpedancia tanto del grupo global como la comparación entro HD y DP se resumen en la tabla 3. Los pacientes en DP tenían más masa grasa, masa celular y masa muscular medida en $\mathrm{Kg}$ totales pero no existían diferencias en los porcentajes.

\begin{tabular}{|l|c|c|c|c|}
\hline & Total $\mathbf{n = 4 2}$ & $\mathbf{H D}=31$ & $\mathbf{D P}=1 \mathbf{1}$ & $\mathbf{p}$ \\
\hline $\begin{array}{l}\text { Angulo } \\
\text { de fase }\end{array}$ & $4,47 \pm 1,57$ & $4,3 \pm 1,7$ & $4,9 \pm 1,1$ & $\mathrm{~ns}$ \\
\hline $\begin{array}{l}\text { ángulo fase } \\
\text { disminuido }\end{array}$ & $60,5 \%$ & $67,7 \%$ & $45,5 \%$ & $\mathrm{~ns}$ \\
\hline $\begin{array}{l}\text { Intercambio Na/ } \\
\text { Kaumentado }\end{array}$ & $1,44 \pm 0,68$ & $1,5 \pm 0,7$ & $1,2 \pm 0,3$ & $\mathrm{~ns}$ \\
\hline $\begin{array}{l}\text { DN intercambio } \\
\text { Na/K }\end{array}$ & $67,4 \%$ & $71 \%$ & $63,6 \%$ & $\mathrm{~ns}$ \\
\hline Masa grasa (Kg) & $19,3 \pm 7$ & $17,9 \pm 7$ & $23,3 \pm 5$ & $\mathrm{P}=0,028$ \\
\hline$\%$ Masa grasa & $29,9 \pm 8,7$ & $29 \pm 9,4$ & $32,2 \pm 6,3$ & $\mathrm{~ns}$ \\
\hline $\begin{array}{l}\text { Masa grasa } \\
\text { disminuida }\end{array}$ & $18,6 \%$ & $25,8 \%$ & 0 & $* *$ \\
\hline BMC & $7,9 \pm 2,6$ & $7,4 \pm 2,6$ & $9,6 \pm 2$ & 0,0017 \\
\hline $\begin{array}{l}\text { Masa celular } \\
\text { (Kg) }\end{array}$ & $19,7 \pm 7,4$ & $18,5 \pm 7,9$ & $23 \pm 7,3$ & $\mathrm{~ns}$ \\
\hline $\begin{array}{l}\% \text { Masa } \\
\text { celular }\end{array}$ & $43,3 \pm 10,2$ & $42 \pm 10,8$ & $47,1 \pm 7,3$ & $\mathrm{~ns}$ \\
\hline $\begin{array}{l}\text { Masa celular } \\
\text { disminuida }\end{array}$ & $34,9 \%$ & $41,9 \%$ & $18,2 \%$ & $\mathrm{~ns}$ \\
\hline $\begin{array}{l}\text { Masa } \\
\text { muscular (Kg) }\end{array}$ & $25,1 \pm 8$ & $23,7 \pm 8,3$ & $29,2 \pm 5,5$ & $\mathrm{P}=0,048$ \\
\hline $\begin{array}{l}\% \text { Masa } \\
\text { muscular }\end{array}$ & $39,3 \pm 9,8$ & $38,9 \pm 10,9$ & $40,4 \pm 5,9$ & $\mathrm{~ns}$ \\
\hline$\%$ TBW & $55,2 \pm 6,5$ & $55,9 \pm 6,7$ & $53 \pm 5,7$ & $\mathrm{~ns}$ \\
\hline $\begin{array}{l}\text { ECW/ } \\
\text { Superficie }\end{array}$ & $11,8 \pm 2,3$ & $12 \pm 2,4$ & $11,3 \pm 2,2$ & $\mathrm{~ns}$ \\
\hline$\%$ ECW & $56,1 \pm 9,6$ & $57,6 \pm 10,1$ & $51,9 \pm 6,9$ & $\mathrm{~ns}$ \\
\hline
\end{tabular}

TBW = agua total corporal.

ECW = agua extracelular

Tabla 3. Datos de bioimpedancia del grupo global y comparación HD vs DP

EI BCM fue superior en DP que en HD. Sólo 8 pacientes, todos en HD, $(18,6 \%)$ presentaron una masa grasa inferior a la que le correspondería para su edad y sexo mientras que $25(58,1 \%)$ tenían exceso de grasa. Un alto porcentaje de pacientes presentaron un ángulo de fase disminuido $(60,5 \%)$ y un intercambio $\mathrm{Na} / \mathrm{K}$ aumentado $(67,4 \%)$ y una disminución del porcentaje de masa celular $(34,9 \%)$ con respecto a los valores normales para su edad y sexo, sin diferencias entre HD y DP. El dato de masa muscular que viene expresado en $\mathrm{Kg}$ se normalizó para la talla en metros, dado que no disponemos de valores en la población normal. La masa muscular en $\mathrm{Kg} / \mathrm{m} 2$ se correlacionó con la edad ( $R=-0,62 p<0,001)$, con el índice de Chalson ( $R=-0,53 p<0,001)$, tiempo en diálisis $(R=-0,038 p=0.01)$ y la creatinina $(R=0,36 y$ $p=0,017)$, $C B(R=0,50 p=0,01)$ y $P M B(R=0,42$ $p=0.006)$. No se relaciona con ingesta de proteínas, ni lípidos; ni con albúmina, prealbumina, Proteina $\mathrm{C}$ Reactiva (PCR) o colesterol. 


\section{Discusión}

Según el criterio que utilicemos para definir la malnutrición su incidencia en diálisis varía mucho $0^{4,5}$. Así, por ejemplo, en nuestro grupo por criterios analíticos como prealbúmina 0 albúmina la incidencia estaría entre un 41,9 y un $53 \%$ respectivamente. Por criterios antropométricos ${ }^{7}$ globales como BMI sería del $26 \%$, mientras que por déficit en grasa corporal total medida por bioimpedancia la incidencia disminuiría al 18,6\%. De acuerdo con los parámetros antropométricos de masa muscular ${ }^{12}$ como el PMB la malnutrición en nuestros pacientes prácticamente no existiría.

Definir la malnutrición en diálisis sigue siendo una asignatura pendiente: la utilización de un único parámetro puede hacer que no detectemos precozmente la malnutrición o que estemos midiendo algo diferente a nutrición; por ejemplo, en el caso de la albúmina existen pacientes en HD con albúminas normales incluso altas y BMI bajos. Por otro lado el uso de índices combinados pueden hacernos confundir malnutrición con comorbilidad o inflamación y que cifremos la malnutrición en diálisis en porcentajes muy altos. Recientemente se ha publicado un documento de consenso de la Sociedad Renal de Nutrición y Metabolismo (ISRNM) ${ }^{19}$ donde se abandona el término de malnutrición para definir esta situación en pacientes en diálisis. Malnutrición significa en términos estrictos: ingesta insuficiente de acuerdo a las necesidades; sin embargo, la situación que a menudo nosotros vemos en los pacientes en diálisis es una alteración del metabolismo (exceso de catabolismo o falta de anabolismo) promovida por citokinas inflamatorias que lleva a la perdida de reservas proteicas y/o energéticas. Por todo ello en ese documento se propone sustituir el término malnutrición por el de "wasting protein-energy"; queda ahora por definir si una intervención nutricional o sobre el anabolismo es capaz de revertirla.

¿Cómo medir la grasa corporal y qué significado tiene en pacientes en diálisis?. Si nos atenemos a los parámetros antropométricos clásicos ${ }^{12}$ encontramos fundamentalmente una desnutrición grasa o calórica, puesto que un alto porcentaje de pacientes en HD (32\%) tienen una disminución del PGT. Esto contrasta con los hallazgos del perímetro de cintura y cadera que indican obesidad abdominal ${ }^{15,16}$ en un $72,1 \%$ de los casos, y con los datos de la bioimpedancia, donde un $51 \%$ de los pacientes tienen exceso de grasa corporal y sólo un $18 \%$ de los pacientes tienen una disminución del porcentaje de masa grasa corporal. Además no encontramos correlación entre el PGT y la masa grasa por bioimpedancia o la RCC, por lo que creemos que el PGT no es un parámetro fiable y debería ser abandonado para definir la malnutrición calórica en diálisis. No podemos olvidar que sigue existiendo un $18 \%$ de pacientes que tienen disminución de masa grasa medida por bioimpedancia ${ }^{9}$ y posiblemente este hecho si sea un marcador importante de malnutrición. En este mismo sentido apuntan los resultados obtenidos con el BMI que es inferior a $23 \mathrm{Kg} / \mathrm{m}^{2}$ en el $26 \%$ de pacientes y tienen una buena correlación con la masa grasa medida por bioimpedancia $(R=0.80)$. La disminución de masa grasa o BMI posiblemente, como ya se ha sugerido en otros trabajos sobre pacientes en diálisis, es un factor de riesgo de mortalidad mayor que la obesidad abdominal o un alto BMI. Nosotros proponemos utilizar el porcentaje de masa grasa medido por bioimpedancia 9 de manera periódica en nuestros paciente ya que nos permite, además de compararnos con la población sana con la misma edad y sexo, detectar de forma precoz pérdidas de grasa ${ }^{8,9}$ en un mismo paciente. En este estudio no encontramos diferencias analíticas o de ingesta corregidas para el peso ideal entre los pacientes con BMI menor de 23 respecto al resto. Tampoco encontramos correlación entre el BMI y la edad, el índice de Chalson o el tiempo en diálisis.

La mayoría de las guías recomiendan la realización de encuestas dietéticas periódicas en los pacientes en diálisis. En nuestro hospital se realizan al mes de inicio de la técnica y por protocolo a los 6 meses y/o cada vez que detectamos alteraciones. Esta práctica nos permite conocer los hábitos alimenticios de los pacientes y corregir déficits mayores y trasgresiones peligrosas como exceso en ingesta de potasio, sodio o fósforo. En este estudio las encuestas dietéticas mostraban que la mayoría de los pacientes tenían ingestas superiores a la $30 \mathrm{Kcal} / \mathrm{kg} / \mathrm{día}$ de calorías y a 1,2 gr/kg/día de proteínas (posiblemente porque se trata de pacientes que llevan tiempo en diálisis y han sido asesorados por una enfermería nefrológica tanto en la fase de prediálisis como posteriormente en diálisis). No observamos diferencias analíticas, antropométricas o en la composición corporal entre 
los pacientes que cumplían las recomendaciones o no. Este hecho estaría en concordancia con lo expresado por la ISRNM de que estos pacientes más que una malnutrición por falta de ingesta como ocurre en la anorexia, lo que tienen es una situación inflamatoria y de déficit de anabolismo. Queda por determinar si altas ingestas, dado que es una situación catabólica, pueden ayudar a frenar o revertir esta situación de "wasting protein-energy"19. En este sentido los hallazgos con pacientes con ingestas superiores a 35 $\mathrm{Kcal} / \mathrm{Kg} /$ día son esperanzadores, ya que si bien puede deberse a que son pacientes más jóvenes y con menos comorbilidad, observamos una composición corporal medida por bioimpedancia más parecida a la población normal.

La BIVA ${ }^{9}$ nos permite conocer mejor la composición corporal y ponerla en relación con población sana de la misma edad y sexo. La mayoría de nuestros pacientes tienen una alteración de la composición corporal consistente fundamentalmente en disminución del ángulo de fase y/o masa celular sana, que todavía no sabemos interpretar bien. Probablemente este midiendo membranas celulares dañadas por la inflamación. Lo importante es que disponemos de medidas objetivas y repetibles en las mismas condiciones de los pacientes lo que nos permitirá ver si las diferentes actuaciones: nutricionales, farmacológicas, cambios en las pautas de diálisis, aumentos de ultrafiltraciones, etc. pueden corregir estas alteraciones de la composición corporal. Otro dato que aporta la BIVA además del porcentaje de masa grasa ya comentado, es la masa muscular medida en $\mathrm{Kg}$ totales y el porcentaje. No disponemos de un valor de porcentaje de masa muscular para la población normal pero si hemos visto que la masa muscular normalizada para la talla mantenía una buena correlación con la edad y el índice de Charlson. Probablemente la masa muscular medida por bioimpedancia y sus cambios nos permitan detectar precozmente el inicio de "wasting proteico" y actuar precozmente.

\section{Conclusiones}

1. Definir malnutrición sigue siendo difícil y según el criterio que utilicemos la incidencia varía mucho.

2. La mayoría de los pacientes presentan un exceso de grasa corporal medida tanto por bio- impedancia como por RCC. No obstante, sigue existiendo un $18 \%$ de pacientes con disminución de masa grasa que coincide con los pacientes con bajo BMI que posiblemente represente un grado avanzado de malnutrición.

3. Las encuesta dietéticas mostraban que la mayoría de los pacientes tenían ingestas superiores a la $30 \mathrm{Kcal} / \mathrm{kg} /$ día y a 1,2 gr/kg/día de proteínas. No observamos diferencias analíticas, antropométricas o en la composición corporal entro los pacientes que cumplían o no las recomendaciones.

4. Mediante BIVA observamos que la mayoría de nuestros pacientes tienen una alteración de la composición corporal consistente fundamentalmente en disminución del ángulo de fase y/o masa celular sana y aumento del intercambio $\mathrm{Na} / \mathrm{K}$; todavía no sabemos interpretar bien estos datos: probablemente estén midiendo membranas celulares dañadas por la inflamación 0 alteraciones en la composición corporal asociadas a la IRC.

\section{Agradecimiento}

Este trabajo ha sido posible gracias a todo el personal de la Unidad de Diálisis de Segovia por su implicación en el proyecto. Gracias a las enfermeras que han dejado constancia escrita de su trabajo, a las auxiliares de enfermería por su esmerado trabajo diario, y a la Dra. $M^{a}$ José Fernández -Reyes por su asesoramiento estadístico y su inestimable colaboración.

\section{Bibliografía}

1. Thunberg BJ, Swamy AP, Cestero RV. Cross-sectional and longitudinal nutritional measurements in maintenance haemodialysis patient. Am J Clin Nut $1981 ; 34: 2005-2012$.

2. Aparicio M, Cano N, Chauveau P et al. Nutritional status of haemodialysis patients: a French national cooperative study. French Study Group for Nutrition in Dialysis. Nephrol Dial Transplant 1999; 14:1679-1686.

3. Ge YQ, Wu ZL, XuYZ, Liao LT. Study on nutritional status of maintenance hemodialysis patients. Clin Nephrol 1998; 50:309-314. 
4. Pifer TB, McCullough KP, Port FK et al. Mortality risk in haemodialysis patients and changes in nutritional indicators:DOPPS. Kidney Int 2002; 62: 2238-2245.

5. Fung F, Sherrard DJ, Gillen DL et al. Increased risk for cardiovascular mortality among malnourished end-stage renal disease patients. Am J Kidney Dis 2002; 40: 307-314.

6. Fernández-Reyes MJ, Álvarez-Ude F, Sánchez R, Mon C, Iglesias $P$, Vázquez A. Estado nutricional comorbilidad e inflamación en hemodiálisis. Nefrología 2000; 20: 540-549.

7. Fouque $D$, Vennegoor M, Wee PT, Wanner C, Basci A, Canaud B, et al. EBPG Guideline on Nutrition. Nephrol Dial Transplant 2007; 22 [Suppl 2]: ii45ii87.

8. Peña Amaro P, García López J. Diplomados en Enfermería. VII premio a la investigación enfermera de la Fundación Iñigo Álvarez de Toledo. 2006.

9. Piccoli A, Negrelli $S$, Caberloto A, Bottazzo S, Rossi B, Pillo L, Maggiore Q. Bivariate normal values of the bioelectrical impedance vector in adult and ederly populations. Am J Clin Nutr, 1995; 61:269-270.

10. Piccoli A, Nescolarde LD, Rossel J, for the Italian CAPD-BIA study group. Bioelectric bioimpedance vector distribution in peritoneal dialysis patients with different hydration status. Kidney Int 2004; 65: 1050-1063.

11. Piccoli A, for the Italian HD-BIA Study Group: identification of operational clues to dry weight prescription in hemodialysis using bioimpedance vectorial analysis. Kidney Int 1998; 53:10361043.

12. Blumenkrantz MJ, Koople JD, Gutman RA, Chan YK, Barbour GL, Robers C, et al: methods for assessing nutritional status of patients with renal failure. Am J. Clin Nutr 1980; 33: 1567-1585.
13. Alatrue Vidal A, Sitges Serra A, Jaurrieta Más $E$, Sitges Creus A. Valoración de los parámetros antropométricos en nuestra población. Med Clin 1982; 78: 404-415.

14. Ricart W, González-Huix F, Conde V y Grup per I’Evaluació de la composició Corporal de la Població de Catalunya. Valoración del estado de nutrición a través de la determinación de los parámetros antropométricos: nuevas tablas de la población laboral de Cataluña. Med Clin 1993; 100:681-691.

15. Soriger Escofet F. La obesidad: Monografía de la Sociedad Española de Endocrinología. Publicado por Ediciones Díaz de Santos, 1994, 55-56.

16. Comité de Expertos de la OMS. El estado físico: uso e interpretación de la antropometría. Ginebra: OMS 1995:387 (Serie de informes técnicos No. 854).

17. Beddhu S, Bruns FJ, Saul M, Seddon P, Zeidel ML. A simple comorbidity scale predicts clinical outcome and cost in dialysis patients. Am J Med 2000; 108(8):609-13.

18. Kopple JD, Zhu X, Lew NL, and E G Lowrie. Body weight-for-height relationships predict mortality in maintenance haemodialysis patients. Kidney Int 1999; 56:1136-1148.

19. Fouque D, Kalantar-Zadeh K, Kopple J, Cano N, Chauveau $P$, Cuppari $L$, et al. A proposed nomenclature and diagnostic criteria for protein -energy wasting in acute and chronic kidney disease. Kidney Int 2008; 73:391-398. 